\title{
$\mathbf{Z}{ }^{\bullet}$ Universtitir Ziekenhuis Gent
}

\section{Physical function measurements predict mortality in ambulatory older men}

Stefanie De Buyser ${ }^{a}$, Mirko Petrovic ${ }^{a}$, Youri Taes ${ }^{b}, K^{2}$ aatje Toye ${ }^{b}$, Jean-Marc Kaufman ${ }^{b}$, Stefan Goemaere ${ }^{b}$

a Department of Geriatrics, ${ }^{b}$ Department of Endocrinology and Unit for Osteoporosis and Metabolic Bone Diseases, Ghent University Hospital, Ghent, Belgium.

Eur J Clin Invest, in press

36 Wintermeeting BVGG / SBGG

Oostende - 22 feb 2013 


\section{Introduction}

- Physical function measurements

$\rightarrow$ health-related outcomes ${ }^{1}$

๑ Functional status measurements

$\leftrightarrow$ objective measurements ${ }^{2}$

- Assessment \& comparison of physical function measurements as predictors for all-cause mortality 


\section{Introduction}

- Timed Up and Go test ${ }^{1}$

๑ falls 2

- mortality ${ }^{3}$

- Evaluation Timed Up and Go test as a predictor for global mortality

- after 15y follow-up

$\odot$ in subjects with underlying comorbidity

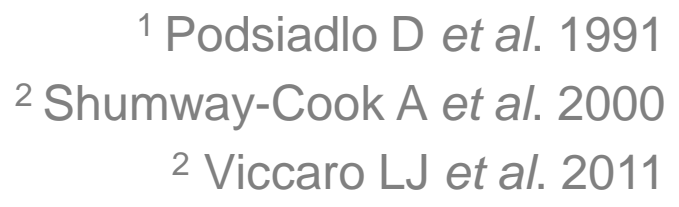




\section{Design}

๑ Observational study (1996 - 2011)

- Community-based setting (Merelbeke)

- Participants:

- 352 ambulatory older men ( / 748)

- aged 70 to 85 at recruitment 


\section{Physical function measurements}

- ADL Rapid disability rating scale-2

- Physical function index <

36-Item short form health survey

- Hand grip strength Muscle strength measurement

๑ Five times sit-to-stand test

- Standing balance

- Timed Up and Go test

Physical performance measurements 


\section{Methods}

๑ Follow-up $>15 y$

- Cox proportional hazard analyses (Z-scores)

- Covariates: age, BMI, smoking status, education, physical activity, and cognitive status

- Comorbidity status:

- cardiovascular disease

- chronic obstructive pulmonary disease

๑ diabetes mellitus 


\section{Results}

๑ 71-86y at baseline

๑ Mean score RDRS-2 $=22$

๑ † 273 / 352 men (78\%)

๑ Median survival time $=\mathbf{1 1 0}$ months 


\section{Results}

Rapid Disability Rating Scale-2 ADL

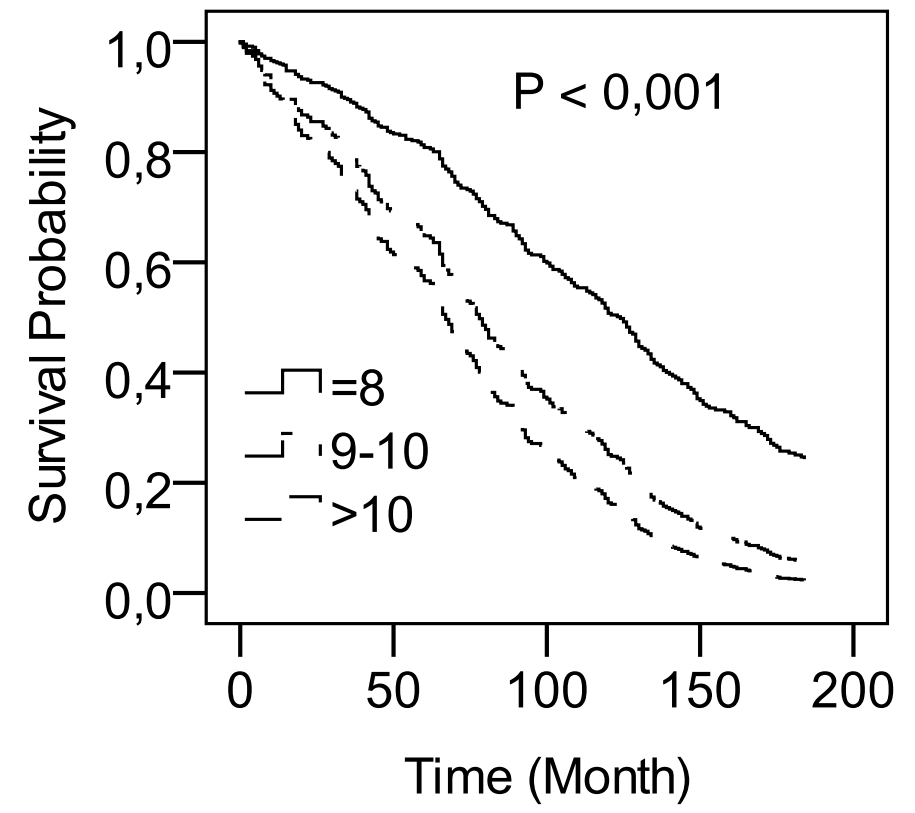

Short Form-36 Physical Function Index

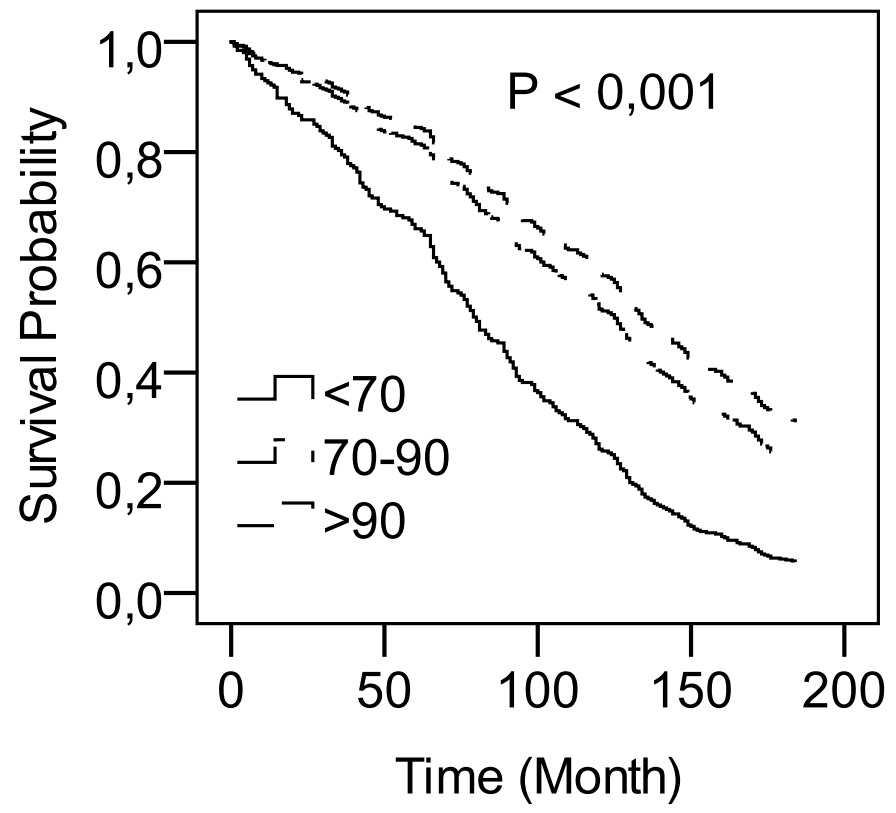




\section{Results}
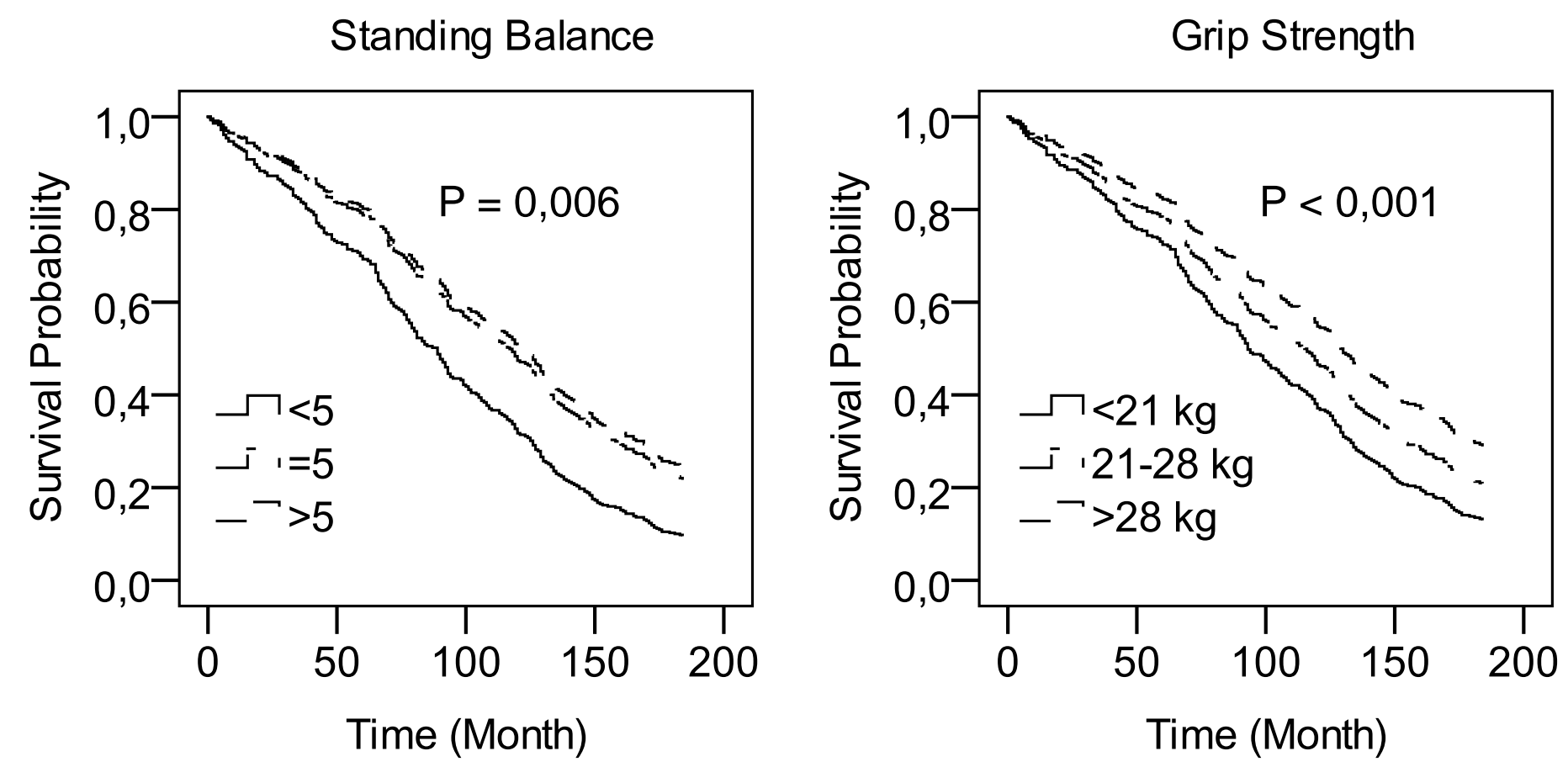


\section{Results}
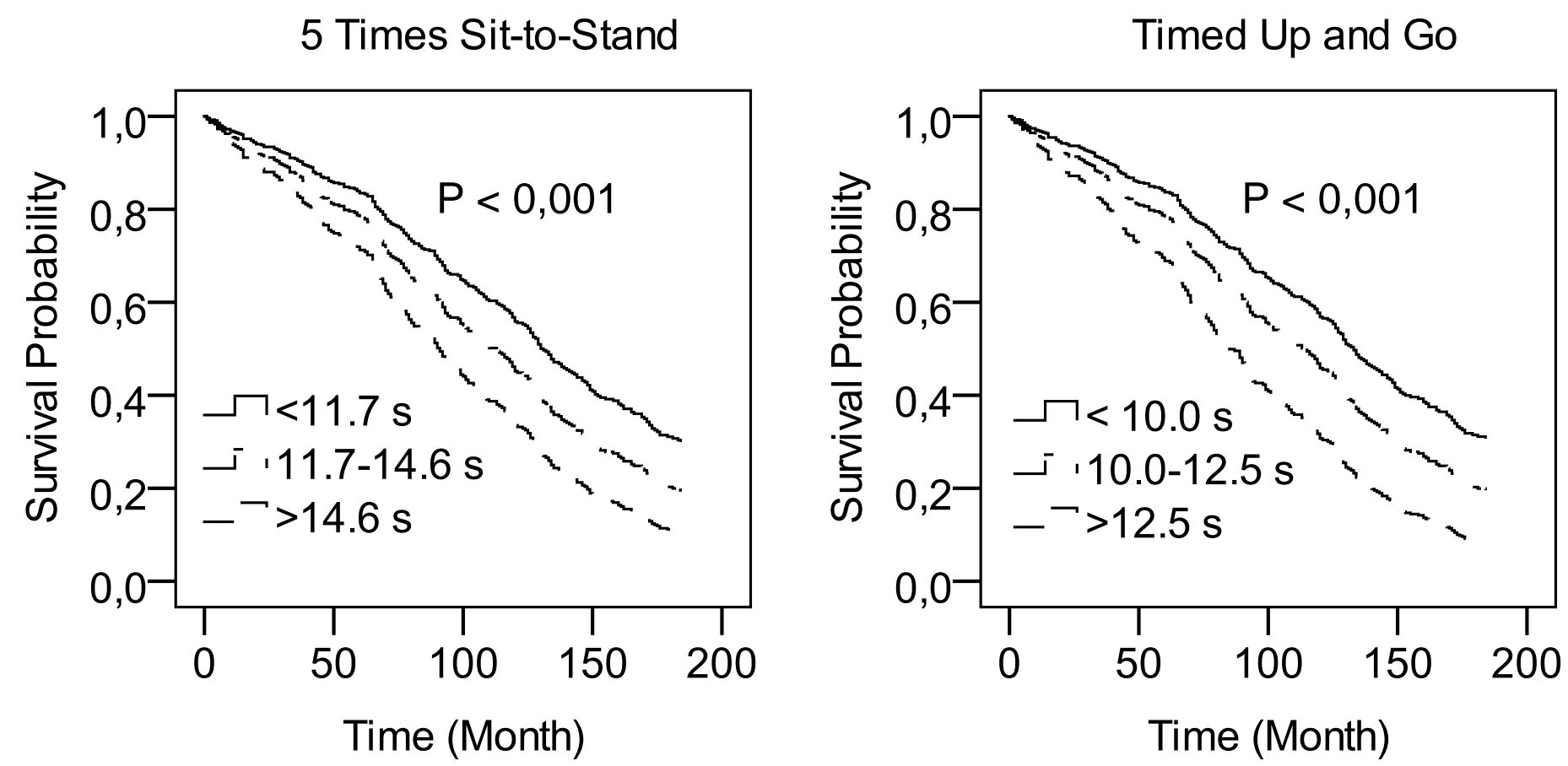


\section{Results}

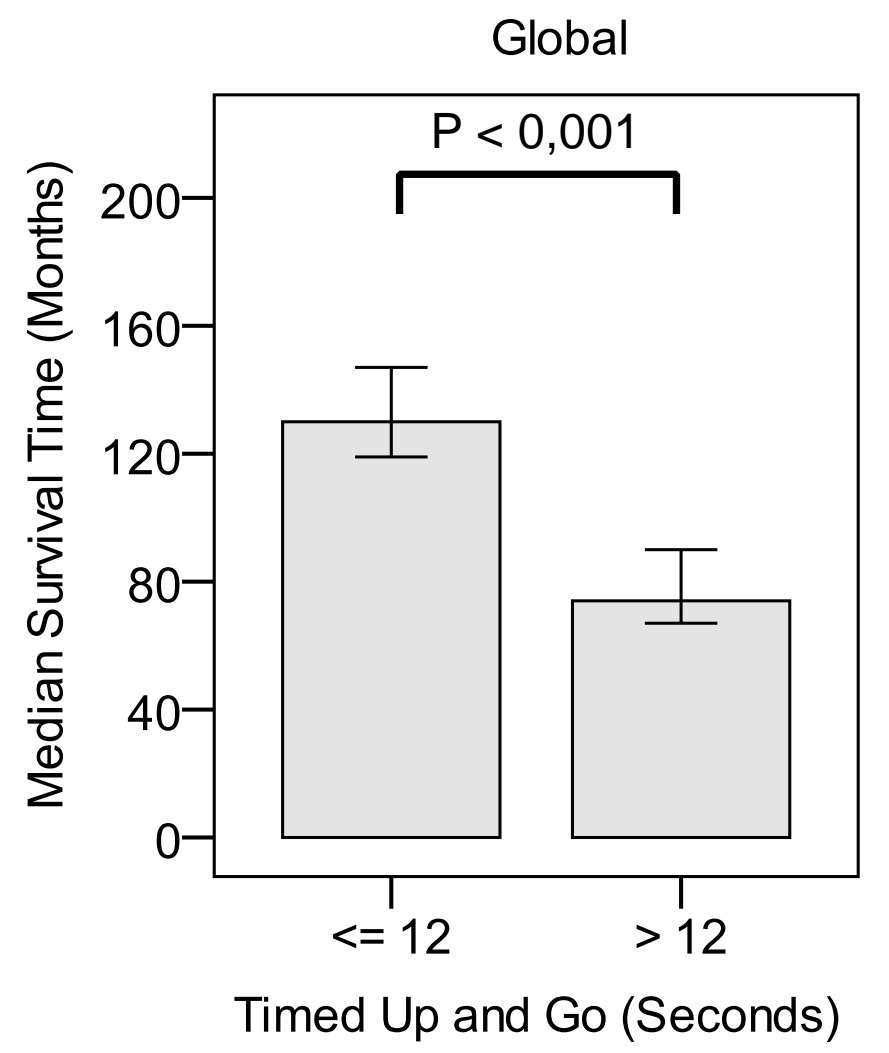

Subjects with Cardiovascular Disease

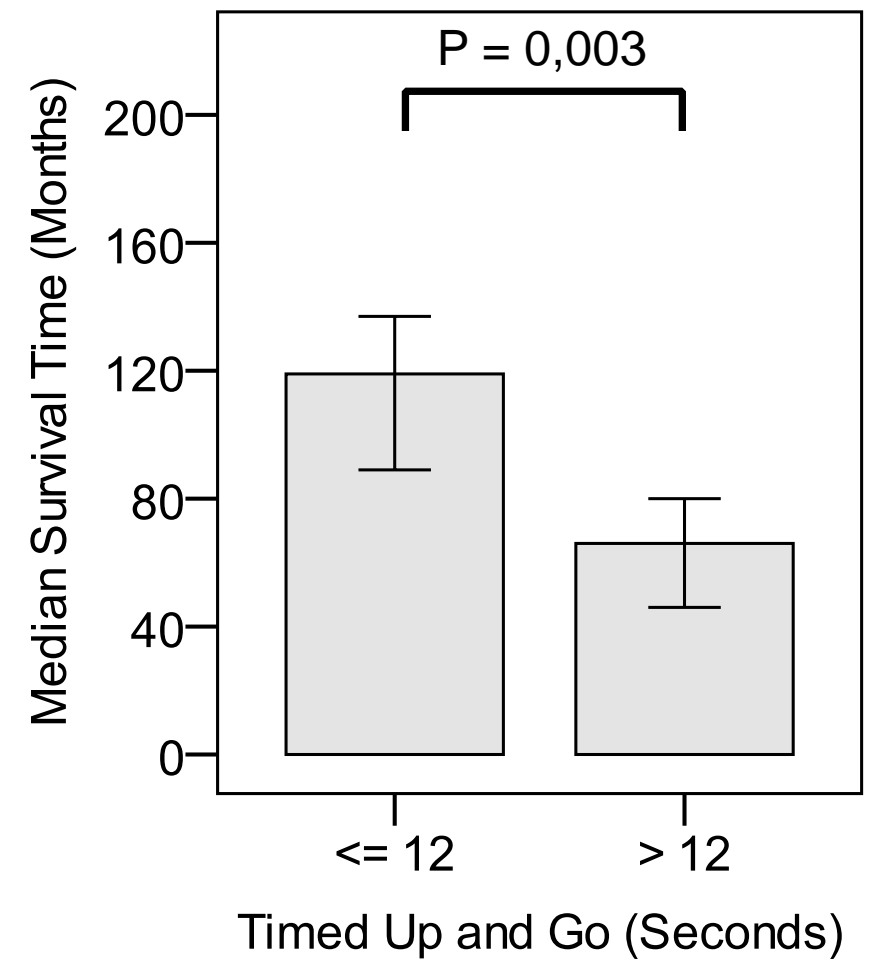




\section{Results}
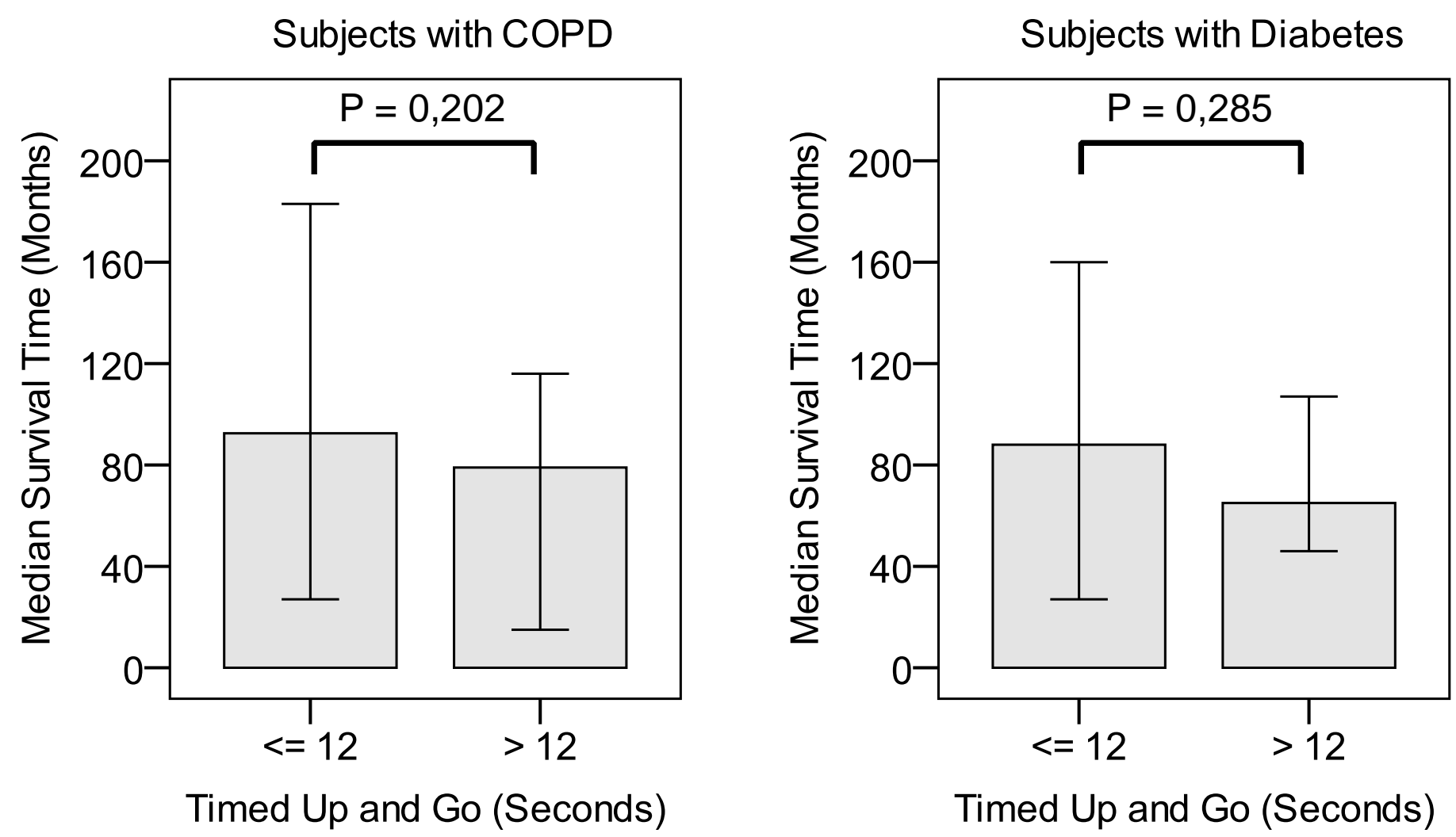


\section{Discussion}

- Weaker association with mortality ${ }^{1}$

\section{$\leftarrow$ Longer follow-up ${ }^{2}$}

- Timed physical performance measures

$\leftrightarrow$ functional status measurements ${ }^{3}$ 


\section{Conclusion}

- Assessment of physical functioning in the evaluation of older persons!

- Timed Up and Go test

- Objective time score

- Quick (mean =12,2 sec)

- No special training

- Feasible (348 / 352)

$\odot$ Little equipment needed 